\title{
Activation of protein expression in electroactive biofilms
}

Daniel A. Phillips ${ }^{1}$, Lina J. Bird², Brian J. Eddie², Matthew D. Yates², Leonard M. Tender², Christopher A. Voigt ${ }^{3}$, and Sarah M. Glaven ${ }^{2 *}$

${ }^{1}$ American Society for Engineering Education, 1818 N Street N.W. Suite 600, Washington DC 20036

${ }^{2}$ Naval Research Laboratory, 4555 Overlook Ave., SW, Washington, DC 20375

${ }^{3}$ Department of Biological Engineering and the Synthetic Biology Center, Massachusetts

Institute of Technology, Cambridge, Massachusetts, USA.

Corresponding author:

Sarah M. Glaven

Sarah.Glaven@nrl.navy.mil

4555 Overlook Ave.

Washington, DC 20375 
Supporting Information

Table of Contents

Supplemental Table 1. Strains, plasmids, and primers.

Supplemental Figure 1. Schematics of three-dimensional design of the electrochemical flow cell.

Supplemental Figure 2. Total electrode biofilm surface coverage and morphology.

Supplemental Figure 3. Current generated from replicate biofilms during growth and induction of YFP expression.

Supplemental Figure 4. Induction of YFP expression within biofilms grown on glass.

Supplemental Figure 5. Transient exposure of electrode biofilms to IPTG images separated by fluorescence signal.

Supplemental Figure 6. Mean fluorescence intensity of YFP and dTomato reporters during growth and induction of YFP expression.

Supplemental Figure 7. Distribution of YFP expression across the electrode surface following continuous and transient induction.

Supplemental Video 1. Electrode biofilm formation over time in the eFC.

Supplemental Video 2. Electrode biofilm formation over time in the eFC and continuous induction of protein expression in early biofilms.

Supplemental Video 3. Electrode biofilm formation over time in the eFC and continuous induction of protein expression in mature biofilms.

Supplemental Video 4. Biofilm formation on cover glass and continuous induction of YFP expression.

Supplemental Video 5. Electrode biofilm formation over time in the eFC and transient induction of protein expression in early biofilms.

Supplemental Video 6. Electrode biofilm formation over time in the eFC and transient induction of protein expression in mature biofilms. 
Supplemental Table 1: Strains, plasmids, and primers used in this study.

\begin{tabular}{|l|l|l|}
\hline Strains & Description & Source \\
\hline $\begin{array}{l}\text { Marinobacter } \\
\text { atlanticus CP1 }\end{array}$ & Wild type Marinobacter strain isolated from MCL biofilm & $\begin{array}{l}\text { Wang et al., } \\
2015^{1}\end{array}$ \\
\hline CP1:dTomato & $\begin{array}{l}\text { M. atlanticus CP1 with dTomato under pLacl promoter inserted in } \\
\text { the genome }\end{array}$ & $\begin{array}{l}\text { Bird et al., } \\
2018^{2}\end{array}$ \\
\hline Plasmids & & \\
\hline pLB36 & YFP under IPTG inducible promoter on pBBr backbone & This Study \\
\hline $\begin{array}{l}\text { pBBR1MCS- } \\
\text { 2::gfpmut3 }\end{array}$ & pBBr1MCS-2 with GFP expressed from constitutive lac promoter & $\begin{array}{l}\text { Bonis and } \\
\text { Gralnick, } \\
2015^{3}\end{array}$ \\
\hline pBBR1MCS-2 & Broad range vector backbone used to create pLB36 & $\begin{array}{l}\text { Kovach et al, } \\
1995^{4}\end{array}$ \\
\hline pAJM336 & Plasmid containing IPTG inducible YFP expression cassette. & $\begin{array}{l}\text { Meyer et al, } \\
2018^{5}\end{array}$ \\
\hline $\begin{array}{l}\text { Primers for } \\
\text { pLB36 } \\
\text { construction }\end{array}$ & Sequence & Description \\
\hline pBBRF3 & gggctcatgagcaaatattttatctgaggtAGCTGTTTCCTGTGTGAAATTG & $\begin{array}{l}\text { Binds } \\
\text { pBBR1MCS- } \\
2\end{array}$ \\
\hline pBBRR2 & acccgcgctcagccaacgatcgttaagcgggGCGTTAATATTTTGTTAAAATTCGC & $\begin{array}{l}\text { Binds } \\
\text { pBBR1MCS- } \\
2\end{array}$ \\
\hline pAJMR3 & ttaacgcgaattttaacaaaatattaacgcCCCGCTTAACGATCGTTGGCTG & $\begin{array}{l}\text { Binds } \\
\text { pAJM336 }\end{array}$ \\
\hline
\end{tabular}

1. Wang, Z.; Leary, D. H.; Malanoski, A. P.; Li, R. W.; Hervey, W. J. t.; Eddie, B. J.; Tender, G. S.; Yanosky, S. G.; Vora, G. J.; Tender, L. M.; Lin, B.; Strycharz-Glaven, S. M., A previously uncharacterized, nonphotosynthetic member of the Chromatiaceae is the primary CO2-fixing constituent in a self-regenerating biocathode. Appl. Environ. Microbiol. 2015, 81 (2), 699-712. 2. Bird, L. J.; Wang, Z.; Malanoski, A. P.; Onderko, E. L.; Johnson, B. J.; Moore, M. H.; Phillips, D. A.; Chu, B. J.; Doyle, J. F.; Eddie, B. J.; Glaven, S. M., Development of a Genetic System for Marinobacter atlanticus CP1 (sp. nov.), a Wax Ester Producing Strain Isolated From an Autotrophic Biocathode. Front. Microbiol. 2018, 9, 3176.

3. Bonis, B. M.; Gralnick, J. A., Marinobacter subterrani, a genetically tractable neutrophilic Fe(II)-oxidizing strain isolated from the Soudan Iron Mine. Front. Microbiol. 2015, 6, 719. 
4. Kovach, M. E.; Elzer, P. H.; Steven Hill, D.; Robertson, G. T.; Farris, M. A.; Roop, R. M.; Peterson, K. M., Four new derivatives of the broad-host-range cloning vector pBBR1MCS, carrying different antibiotic-resistance cassettes. Gene 1995, 166 (1), 175-176.

5. Meyer, A. J.; Segall-Shapiro, T. H.; Glassey, E.; Zhang, J.; Voigt, C. A., Escherichia coli "Marionette" strains with 12 highly optimized small-molecule sensors. Nat. Chem. Biol. 2018. 

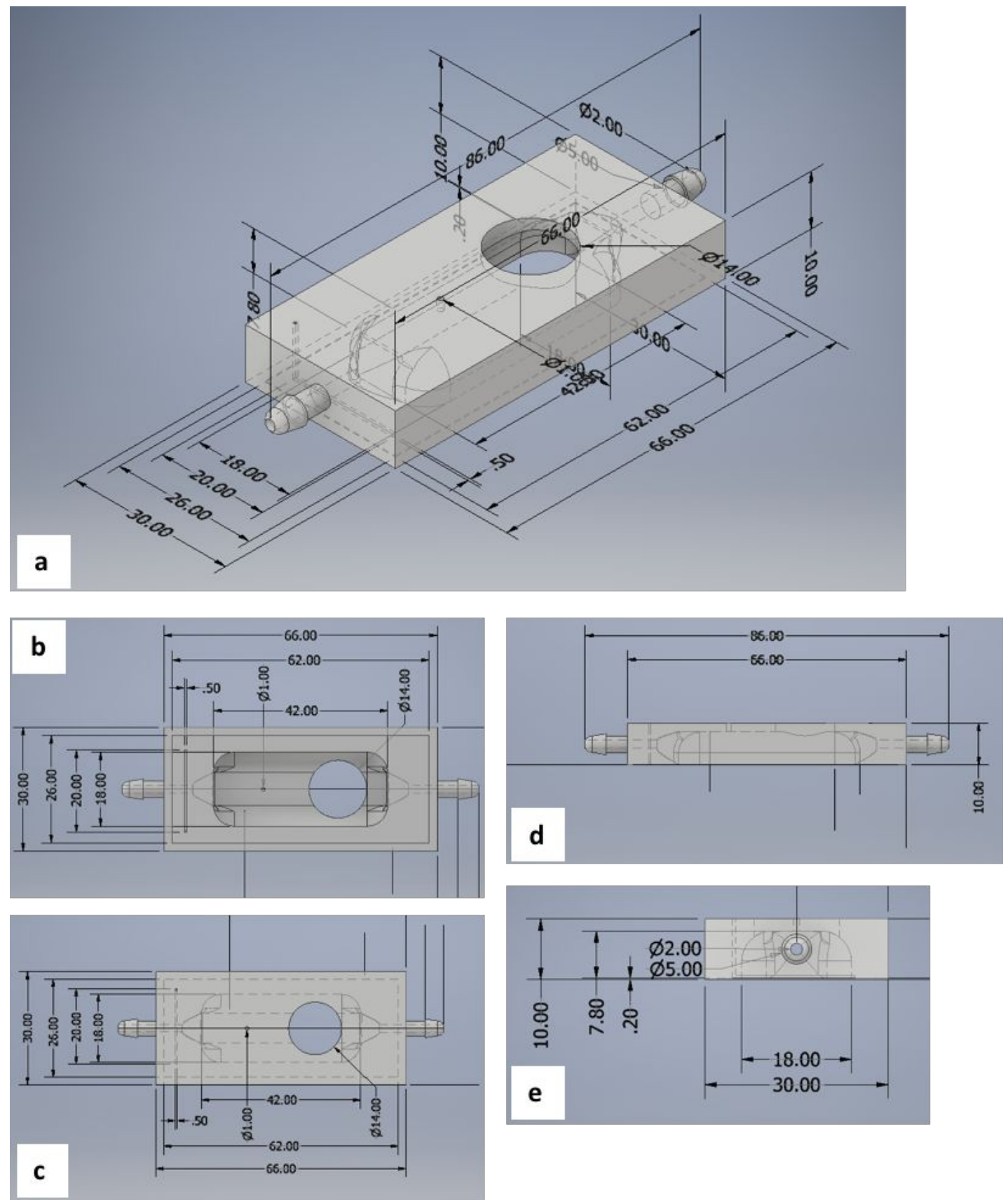

Supplemental Figure 1. Schematics of three-dimensional design of the electrochemical flow cell. a. Profile view of the overall electrochemical flow cell (eFC) CAD design (Autodesk Inventor). b.-d. Side view, top down, and bottom view of the electrochemical flow cell design. e. Front view of the eFC outlet. All values are in millimeters $(\mathrm{mm})$. 

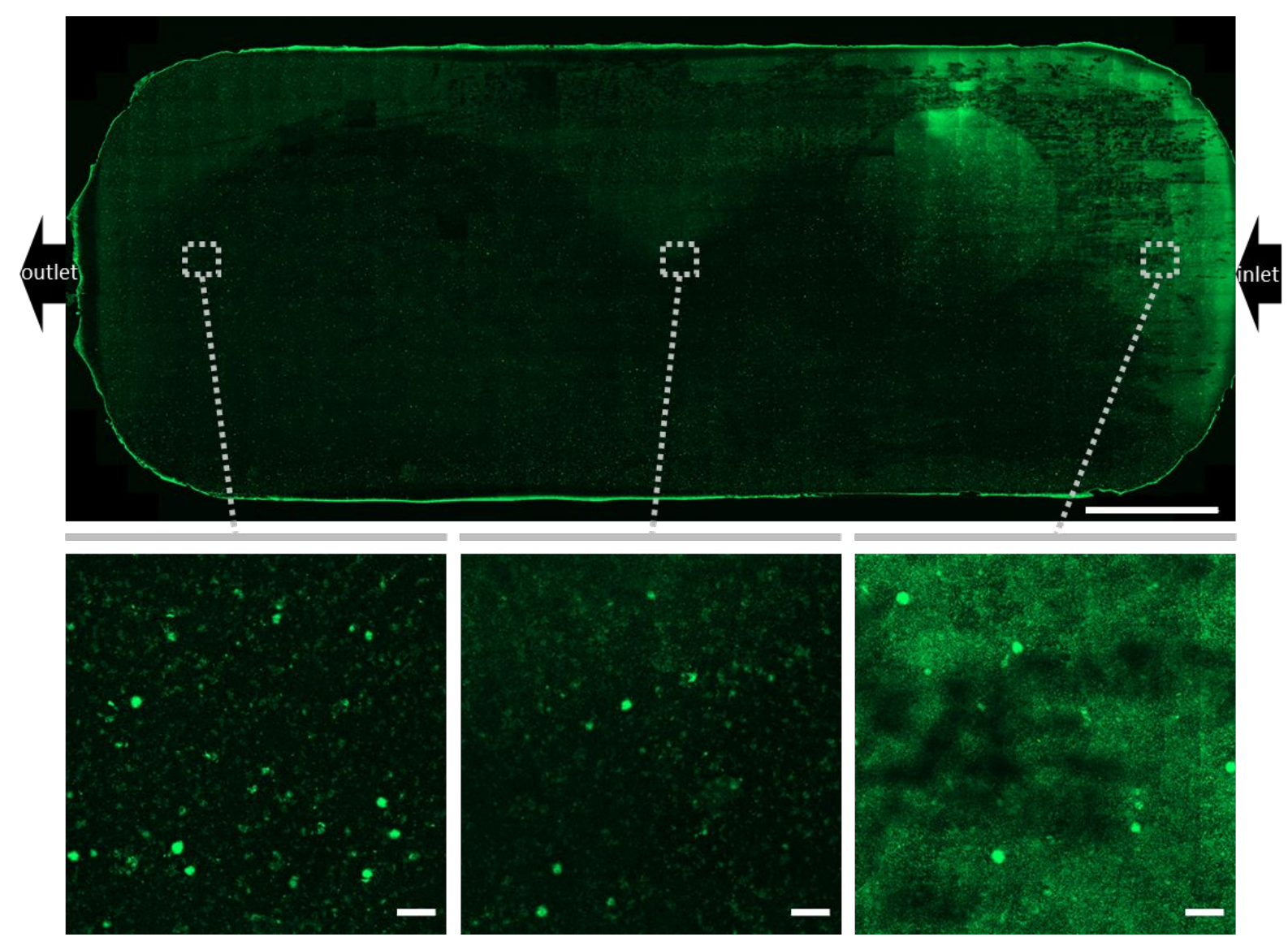

Supplemental Figure 2. Total electrode biofilm surface coverage and morphology. All images are maximum intensity projections of electrode grown (510 mV vs. SHE) M. atlanticus biofilms constitutively expressing GFP. The top panel represents a composite image of a biofilm after 48 h of growth (scale bar $=5000 \mu \mathrm{m}$ ). Enlarged regions near the inlet port (right), middle interior (middle), and outlet port (left) are indicated by the white squares (scale bar $=100 \mu \mathrm{m}$ ). 
a

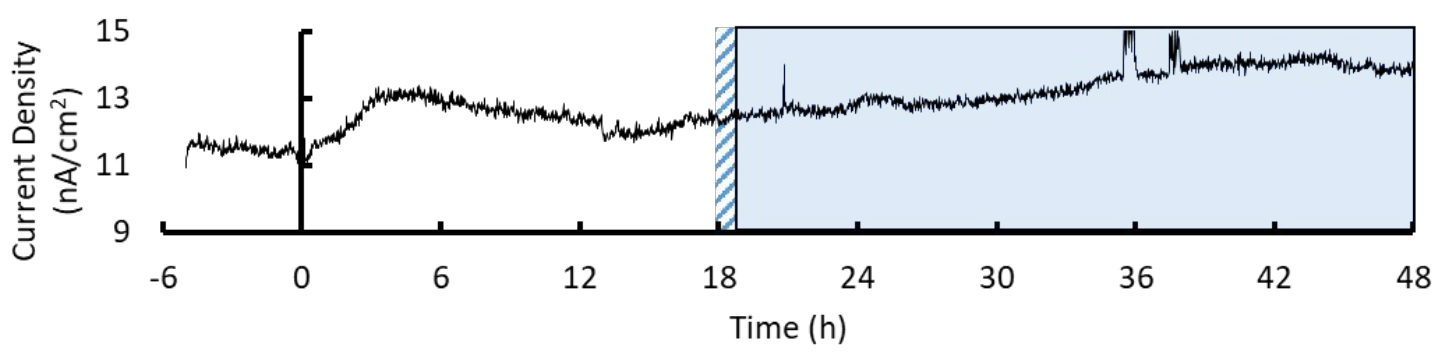

b

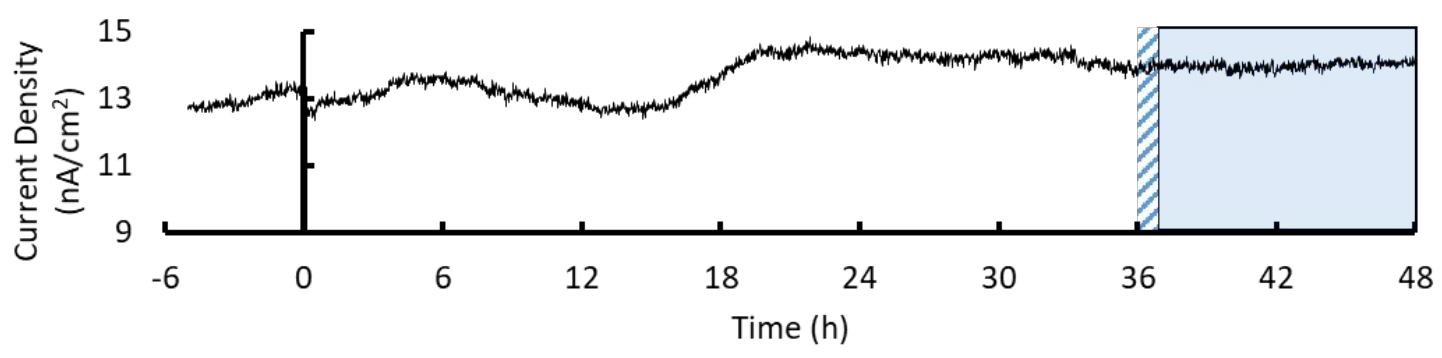

c

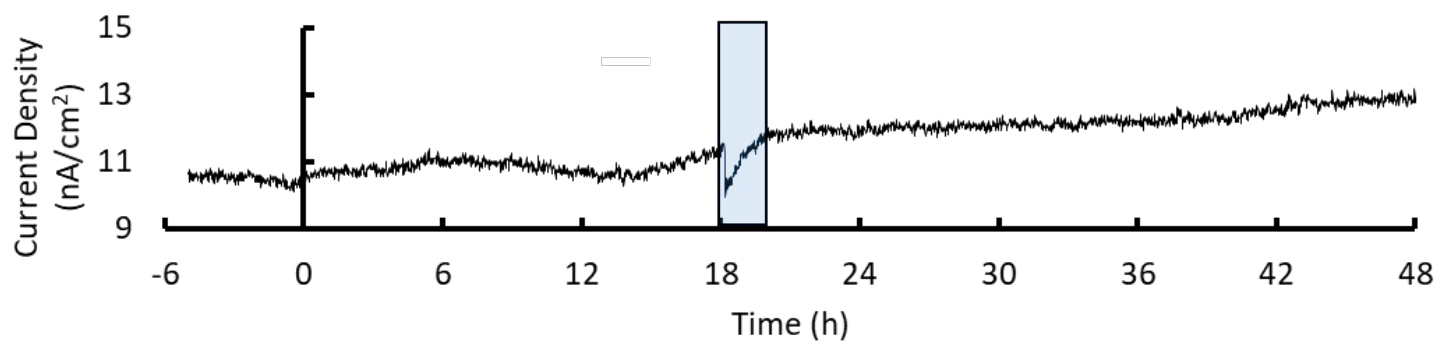

d

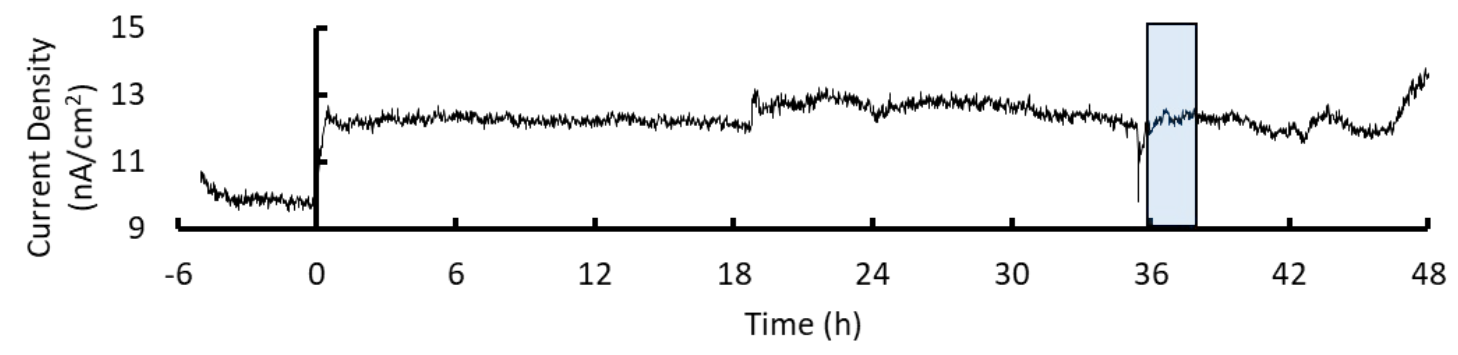

Supplemental Figure 3. Current generated from replicate biofilms during growth and induction of YFP expression. Addition of IPTG to either the medium reservoir is indicated as dashed blue lines. Onset of biofilm exposure to IPTG is represented by the blue shaded area, and the width of the shaded area signifies period of exposure to IPTG. During continuous exposure, IPTG is calculated (Methods) to arrive $1 \mathrm{~h}$ after IPTG is added to the medium reservoir. Retention time of IPTG within the eFC chamber following direct injection for transient exposure is calculated to be $2 \mathrm{~h}$. a.-d. Chronoamperometry (510 mV vs. SHE) was recorded from biofilms constitutively expressing dTomato and induced to express YFP in response to IPTG (500 $\mu \mathrm{M})$. a. Continuous exposure beginning at 18 h. b. Continuous exposure beginning at 36 h. c. Transient exposure at 18h. d. Transient exposure at $36 \mathrm{~h}$. 


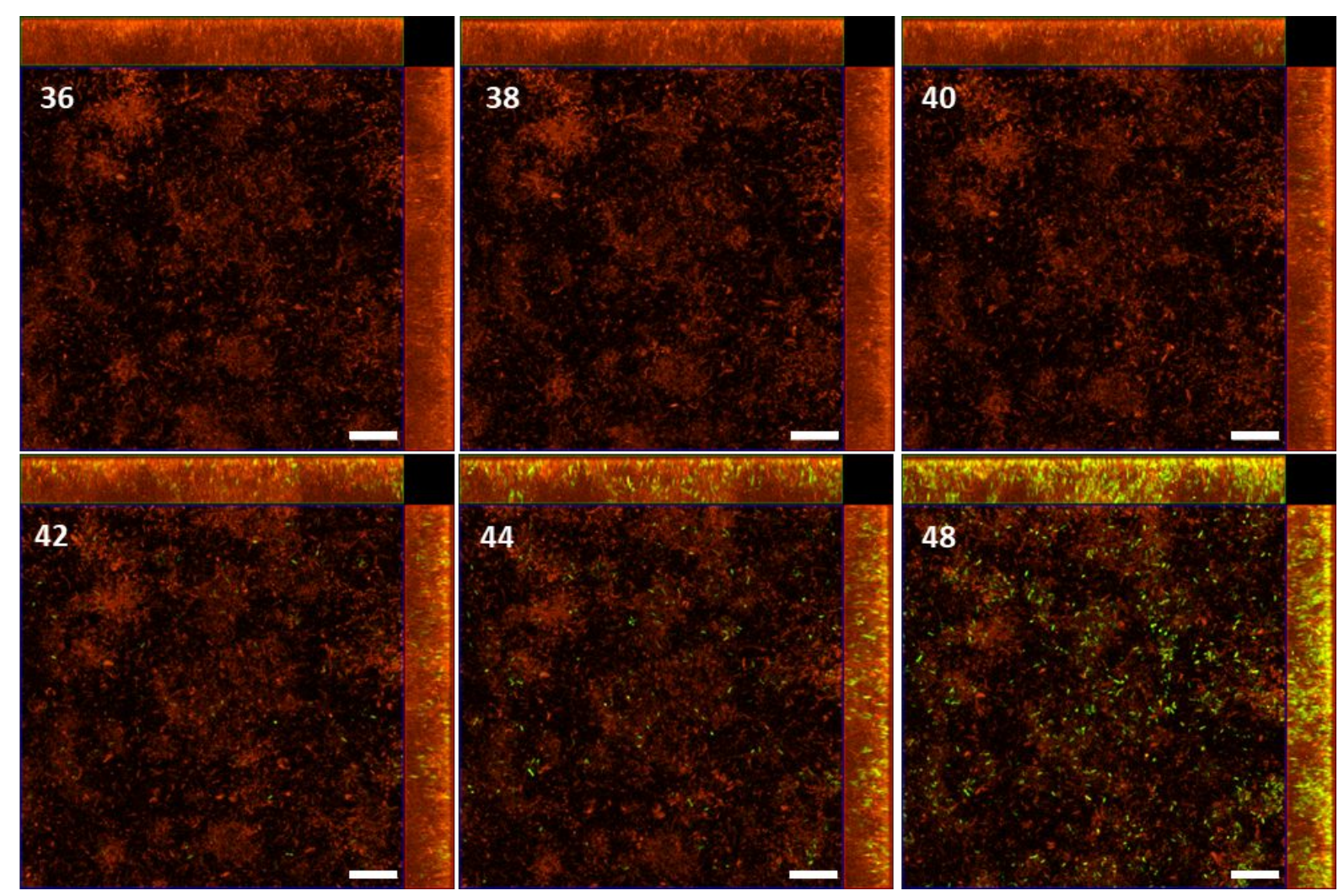

Supplemental Figure 4. Induction of YFP expression within biofilms grown on glass. Images represent top down (center) and side view (top and side panels) maximum intensity projections of $M$. atlanticus biofilms grown on uncoated cover glass over time within the eFC. Cells constitutively express dTomato (red) and YFP (green) is expressed in response to continuous exposure to IPTG $(500 \mu \mathrm{M})$ starting at $36 \mathrm{~h}$. IPTG is calculated (Methods) to arrive at the biofilm $1 \mathrm{~h}$ after it is added to the medium reservoir. Scale bar $=20 \mu \mathrm{m}$. 
a

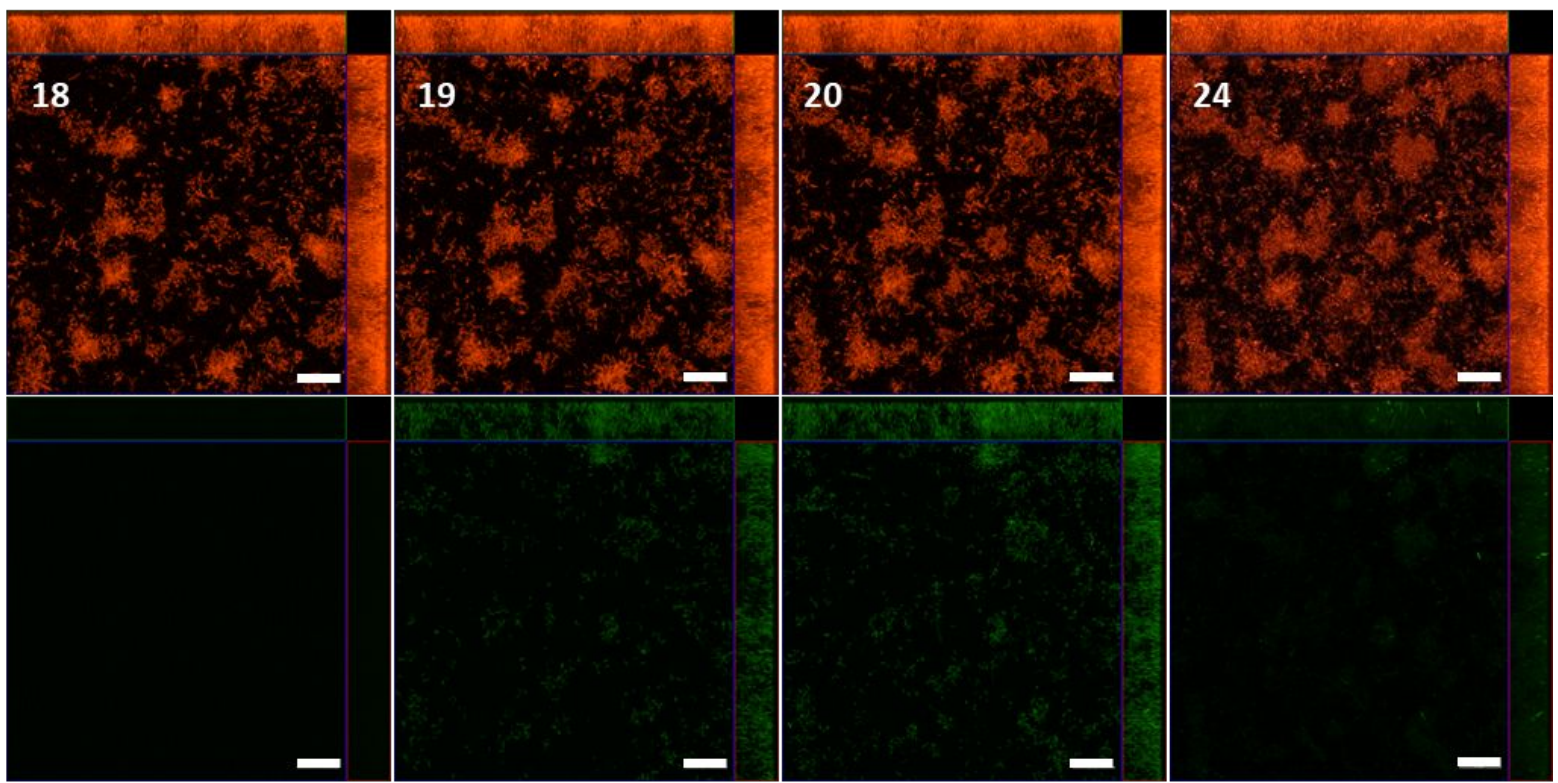

b

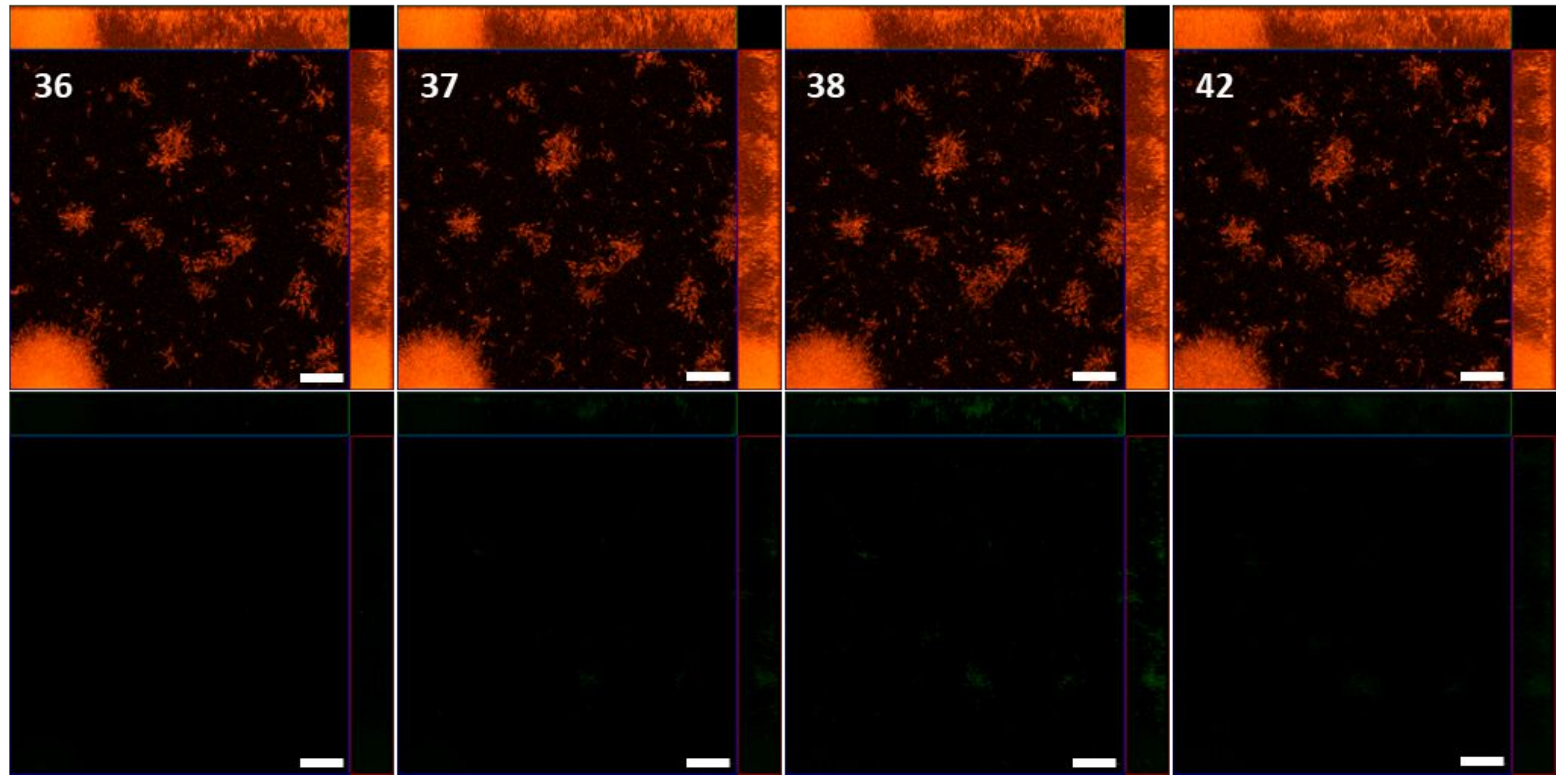

Supplemental Figure 5. Transient exposure of electrode biofilms to IPTG images separated by fluorescence signal. a.-b. Image panels are those depicted in main text Figure 5 and separated here by fluorescence emission spectra. Images represent top down (center) and side view (top and side panels) maximum intensity projections of $M$. atlanticus biofilms grown on ITO electrodes (510 mV vs. SHE) constitutively expressing dTomato (red). Cells are induced to express YFP (green) within the eFC by transient exposure to IPTG through the in-line inoculation port. a. Transient exposure at 18h. b. Transient exposure at 36h. Retention time of IPTG within the eFC chamber following direct injection is calculated (Methods) to be $2 \mathrm{~h}$. Scale bar $=20 \mu \mathrm{m}$. 

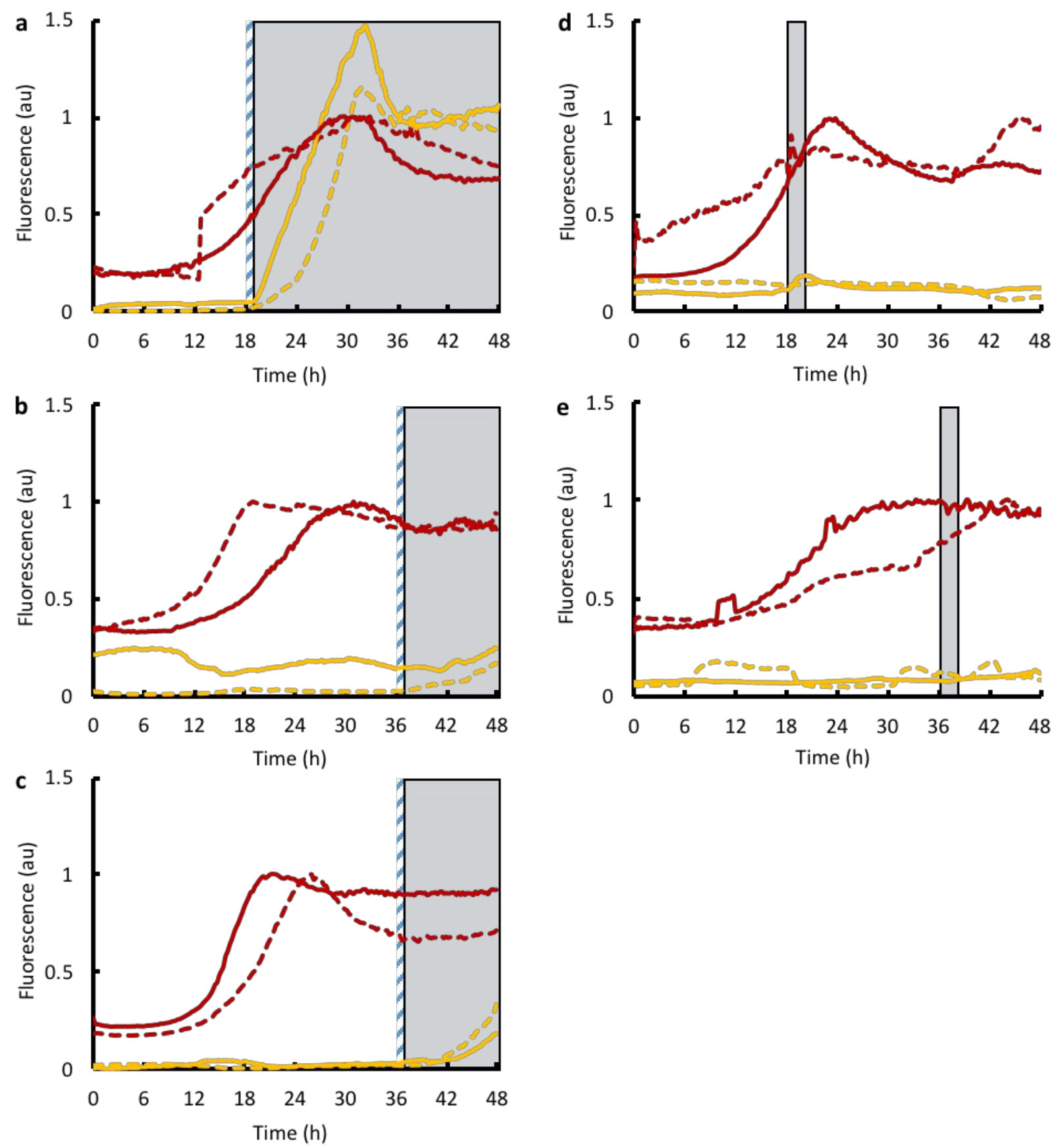

Supplemental Figure 6. Mean fluorescence intensity of YFP and dTomato reporters during growth and induction of YFP expression. Mean fluorescence at each time point for YFP (yellow) and dTomato (red) was scaled relative to the maximum intensity of dTomato for each experiment. Blue hashed boxes indicate time between adding IPTG to medium reservoir and arrival within the eFC chamber, and blue boxes indicate presence of IPTG within the eFC chamber. Solid lines represent the experiments shown in Figures 4 and 5, and Supplemental Figure 3. Dashed lines represent replicate experiments performed to verify reproducibility. a. Continuous induction starting at $18 \mathrm{~h}$ (Figure 4a, Supplemental Video 2). b. Continuous induction starting at $36 \mathrm{~h}$ (Figure 4b, Supplemental Video 3). c. Continuous induction starting at 
$36 \mathrm{~h}$ on glass slides (Supplemental Figure 4, Supplemental Video 4). d. A single pulse of IPTG at $18 \mathrm{~h}$ (Figure 5a, Supplemental Video 5). e. A single pulse of IPTG at $36 \mathrm{~h}$ (Figure 5b,

Supplemental Video 6).

a
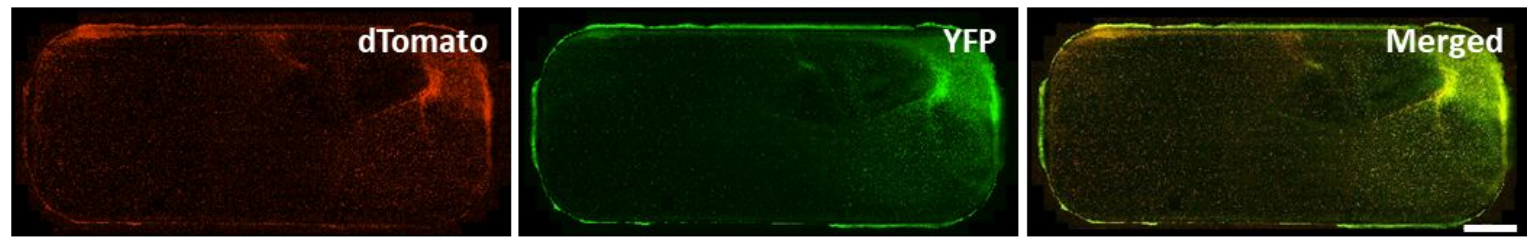

b
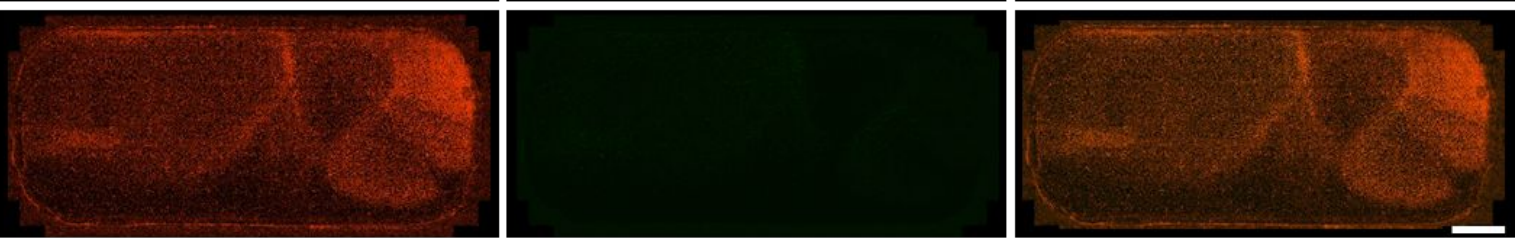

c
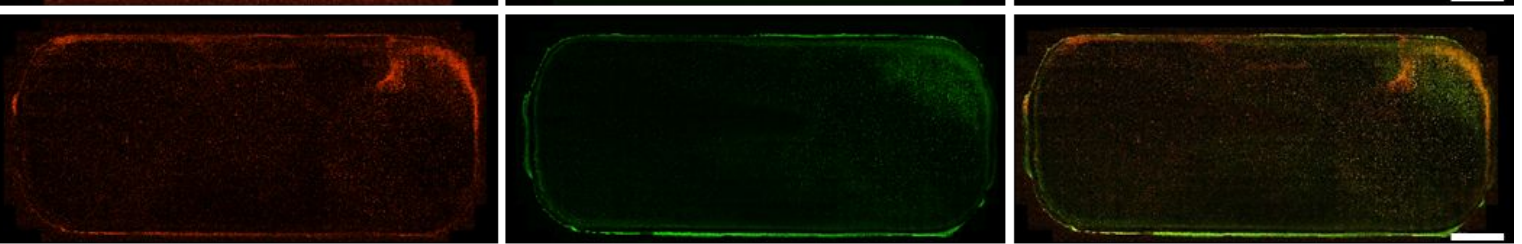

d
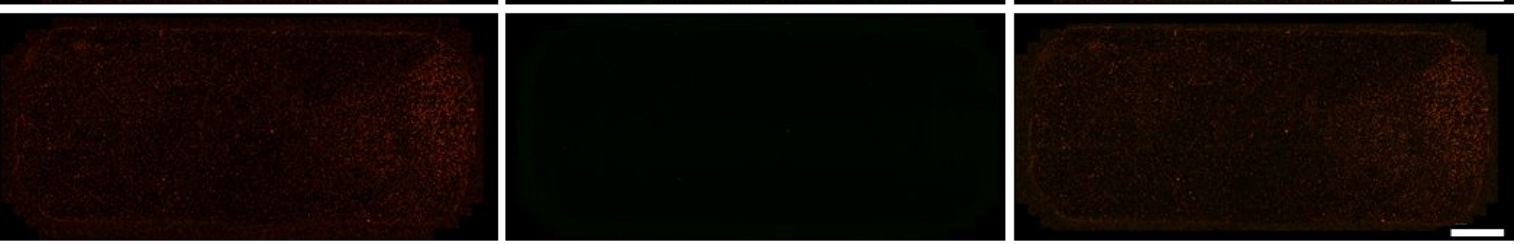

e
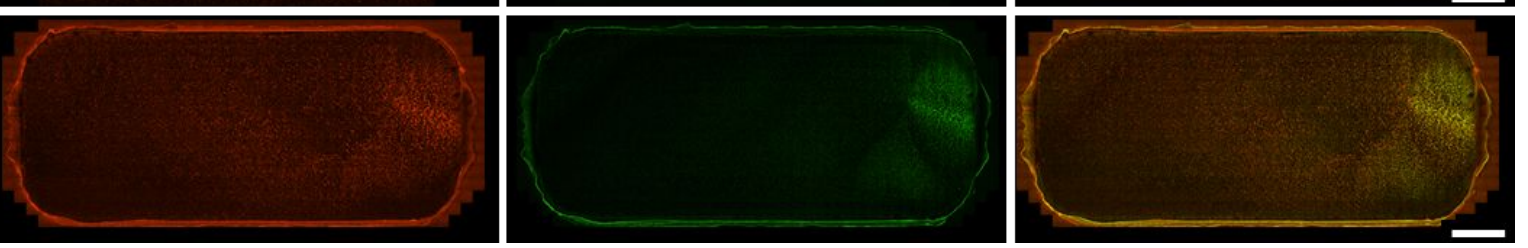

Supplemental Figure 7. Distribution of YFP expression across the electrode surface following continuous and transient induction. a.-e. All panels are composite images of maximum intensity projections of electrode grown ( $510 \mathrm{mV}$ vs. SHE) M. atlanticus biofilms. Panels correspond to time course imaging of biofilms depicted in main text figure and supplemental figures and videos as indicated. Images represent the entire electrode surface after $48 \mathrm{~h}$ within the eFC. Cells constitutively express dTomato (red), and YFP (green) is expressed in response to exposure to IPTG $(500 \mu \mathrm{M})$ a. Continuous exposure at $18 \mathrm{~h}$ (Figure 4a and Supplemental Video 2). b. Transient exposure at $18 \mathrm{~h}$ (Figure 5a, Supplemental Video 5). c. Continuous exposure at $36 \mathrm{~h}$ (Figure 4b, Supplemental Video 3). d. Transient exposure at $36 \mathrm{~h}$ (Figure 5b, Supplemental Video 6). e. Continuous exposure of biofilms grown on glass at $36 \mathrm{~h}$ (Supplemental Figure 3, Supplemental Video 4). Scale bar $=5000 \mu \mathrm{m}$. 


\section{Supplemental Videos}

Supplemental Video 1. Electrode biofilm formation over time in the eFC. Time course video (15 min / frame, 5 frames / second) of maximum intensity projections of $M$. atlanticus biofilms formed on ITO electrodes (510 mV vs. SHE) constitutively expressing GFP. Video represents imaging over the course of $48 \mathrm{~h}$. Corresponding current measured from this biofilm is in Figure 2a. Scale bar $=20 \mu \mathrm{m}$.

Supplemental Video 2. Electrode biofilm formation over time in the eFC and continuous induction of protein expression in early biofilms. Time course video (15 min / frame, 5 frames / second) of maximum intensity projections of $M$. atlanticus biofilms formed on ITO electrodes (510 mV vs. SHE) constitutively expressing dTomato (red) and induced to express YFP (green) following continuous exposure to IPTG starting at $18 \mathrm{~h}$. Video represents imaging over the course of $48 \mathrm{~h}$. Corresponding current measured from this biofilm is in Figure 4a. Scale bar $=20$ $\mu \mathrm{m}$.

Supplemental Video 3. Electrode biofilm formation over time in the eFC and continuous induction of protein expression in mature biofilms. Time course video (15 $\mathrm{min} / \mathrm{frame}, 5$ frames / second) of maximum intensity projections of $M$. atlanticus biofilms formed on ITO electrodes (510 mV vs. SHE) constitutively expressing dTomato (red) and induced to express YFP (green) following continuous exposure to IPTG starting at $36 \mathrm{~h}$. Video represents imaging over the course of $48 \mathrm{~h}$. Corresponding current measured from this biofilm is in Figure $4 \mathrm{~b}$. Scale bar $=20 \mu \mathrm{m}$.

Supplemental Video 4. Biofilm formation on coverglass and continuous induction of YFP expression. Time course video ( 5 frames / second) of maximum intensity projections of $M$. atlanticus biofilms grown on coverglass constitutively expressing dTomato (red) and induced to express YFP (green) following continuous exposure to IPTG starting at $36 \mathrm{~h}$. Video represents imaging over the course of $48 \mathrm{~h}$. Still images from this biofilm are in Figure S3. Scale bar $=20$ $\mu \mathrm{m}$.

Supplemental Video 5. Electrode biofilm formation over time in the eFC and transient induction of protein expression in early biofilms. Time course video ( 5 frames / second) of maximum intensity projections of $M$. atlanticus biofilms formed on ITO electrodes ( $510 \mathrm{mV}$ vs. $\mathrm{SHE}$ ) constitutively expressing dTomato (red) and induced to express YFP (green) following transient exposure to IPTG starting at $18 \mathrm{~h}$. Video represents imaging over the course of $48 \mathrm{~h}$. Corresponding current measured from this biofilm is in Figure 5a. Scale bar $=20 \mu \mathrm{m}$.

Supplemental Video 6. Electrode biofilm formation over time in the eFC and transient induction of protein expression in mature biofilms. Time course video ( 5 frames / second) of maximum intensity projections of $M$. atlanticus biofilms formed on ITO electrodes (510 $\mathrm{mV}$ vs. SHE) constitutively expressing dTomato (red) and induced to express YFP (green) following transient exposure to IPTG starting at $36 \mathrm{~h}$. Video represents imaging over the course of $48 \mathrm{~h}$. Corresponding current measured from this biofilm is in Figure 5b. Scale bar $=20 \mu \mathrm{m}$. 